\title{
Generadores de valor del proceso logístico de las empresas floricultoras de Cundinamarca
}

\section{Value generators of the logistic process of the flower growers of Cundinamarca}

DOI: https://doi.org/10.17981/econcuc.41.2.2020.Org.2

Artículo de investigación.

Fecha de recepción: 21/11/2019

Fecha de devolución: 17/02/2020

Fecha de aceptación: 13/03/2020

Fecha de publicación: 17/03/2020

Wilfrido Javier Arteaga Sarmiento Universidad Militar Nueva Granada. Bogotá, D.C. (Colombia) wilfrido.arteaga@unimilitar.edu.co

\author{
Abraham Jesús González \\ Universidad Militar Nueva Granada. \\ Cajicá (Colombia) \\ abraham.gonzalez@unimilitar.edu.co
}

\section{José Ignacio Rodríguez Molano \\ Universidad Distrital Francisco José de Caldas. Bogotá, D.C. (Colombia) jirodriguez@udistrital.edu.co}

\section{Para citar este artículo:}

Arteaga, W., González, A. y Rodríguez, J. (2020). Generadores de valor del proceso logístico de las empresas floricultoras de Cundinamarca. Económicas CUC, 41(2), 29-48. DOI: https://doi. org/10.17981/econcuc.41.2.2020.Org.2

\section{Resumen}

Una organización requiere suministrar bienes y servicios a los clientes, cuándo, cómo y dónde los requieran; ello se logra, por medio de actividades de valor, enmarcadas dentro del proceso logístico. Bajo este contexto surgió la presente investigación, con el propósito de determinar cuáles son los elementos que impulsan el valor en las actividades del proceso logístico de las empresas del sector floricultor del departamento de Cundinamarca. El estudio se tipificó como descriptivo, no experimental y transversal. La información fue recolectada a través de dos cuestionarios, uno para los clientes internos del proceso logístico y otro para los trabajadores del área logística. La confiabilidad se obtuvo a través del método Alfa de Cronbach y los valores resultantes para los cuestionarios aplicados fueron: clientes de 0.797 y trabajadores logísticos de 0.877 ; los cuales son altamente confiables. Una vez aplicados y analizados los instrumentos, se evidenció que la mayoría de los factores que generan valor en las actividades del proceso logístico son coincidentes entre las poblaciones estudiadas y conservan relación con las bases teóricas utilizadas. Se concluye que el éxito de una organización no solo es consecuencia lógica de realizar bien las acciones operativas de apoyo, sino que además es necesario generar valor en cada actividad de la empresa para satisfacer los requerimientos de los clientes y mantenerlos en el tiempo.

Palabras clave: Generadores de valor; proceso logístico; actividades de valor; cadena de valor; sector floricultor; Cundinamarca

\begin{abstract}
An organization requires supplying goods and services to customers, when, how and where they require them; This is achieved through valuable activities, framed within the logistics process. In this context, this research emerged, with the purpose of determining what are the elements that drive value in the activities of the logistics process of companies in the floriculture sector of the department of Cundinamarca. The study was classified as descriptive, non-experimental and cross-sectional. The information was collected through two questionnaires, one for internal clients of the logistics process and another for workers in the logistics area. Reliability was obtained through the Cronbach's Alpha method and the resulting values for the applied questionnaires were: clients of 0.797 and logistics workers of 0.877 ; which are highly reliable. Once the instruments were applied and analyzed, it was evident that most of the factors that generate value in the activities of the logistics process are coincident among the populations studied and are related to the theoretical bases used. It is concluded that the success of an organization is not only a logical consequence of performing well the operational support actions, but also it is necessary to generate value in each activity of the company to satisfy the requirements of the clients and maintain them over time. Keywords: Value generators; logistics process; valuable activities; value chain; floriculture sector; Cundinamarca.
\end{abstract}




\section{INTRODUCCIÓN}

Actualmente, las organizaciones afrontan fluctuaciones frecuentes de forma global, tanto a nivel nacional como internacional, por lo cual el proceso de desarrollo empresarial, los avances tecnológicos y las comunicaciones; deben estar intrínsecas en las mismas para mantener sus ventajas competitivas y así prevalecer en el mercado. La relevancia de la logística en las organizaciones es cada día mayor, sin importar su tamaño, pues es un elemento básico de su operación, necesario para mejorar la competitividad y productividad.

Partiendo de dicha premisa, sobre todo las empresas del sector productivo deben estar inmersas en una mejora continua para que de esta manera su sistema de gestión sea lo suficientemente eficaz para satisfacer las necesidades de la demanda. Sin embargo, Bowersox, Cross, \& Cooper (2007), Chumaceiro, Chirinos y Reyes (2016) y Paredes-Chacín (2017) afirman que, en los últimos años, cada vez más las organizaciones reconocen que el éxito depende de la perspectiva de valor; esto significa personalizar los servicios de valor agregado, más allá del producto y posicionamiento, con el fin de alcanzar una verdadera diferencia para los clientes.

Aunado a lo antes mencionado, es importante señalar la cadena de valor; la cual es una herramienta ideada por Porter (2015) basada en un método de análisis estratégico. Su objetivo principal es proporcionar una clara comprensión de lo que es una estructura lógica del negocio, estableciendo la fuente de ventaja competitiva, identificando las oportunidades de cambio organizacional y de negocios, para finalmente generar una herramienta de comunicación, así como un marco de referencia gerencial.

En este orden de ideas, Casanovas \& Cuatrecasas (2003) corroboran esta correspondencia significativa entre el valor y la logística, avalando la idea de que el objetivo de la logística consiste en satisfacer la demanda de los clientes eficazmente y optimizando su costo. Se debe considerar, además, que los productos y/o servicios sean adecuados, estén en los lugares pertinentes, en el momento preciso, así como en las condiciones exigidas por el cliente. Estos últimos supuestos son basamentos de un adecuado proceso logístico.

El presente artículo de investigación buscará determinar cuáles son los elementos que impulsan el valor en las actividades del proceso logístico de las empresas del sector floricultor del departamento de Cundinamarca.

\section{ACERCAMiEnTo TEÓRICO}

Según Bowersox, Cross \& Cooper (2007) el valor logístico se consigue cuando las organizaciones son capaces de hacer coincidir su capacidad operativa, con las expectativas y los requerimientos de los clientes. Un proceso logístico bien estructurado permite un mayor impacto en los clientes, al mismo tiempo de permitir controlar las variaciones operativas y minimizar el compromiso del inventario, siendo lo más importante la relevancia del accionar logístico para los clientes específicos (Bowersox, Cross \& Cooper, 2007). 
Por su parte, considera que la logística está para crear valor a los clientes, proveedores y accionistas de las organizaciones. Además, que este valor logístico está expresado en términos de tiempo y lugar, pues el bien o servicio no tiene valor a menos que esté en manos de los clientes cuando y donde ellos deseen consumirlos. Mientras que López (2019) y Ramírez, Valero y Martínez (2019) plantean que el valor de las actividades logísticas viene expresado en términos de tiempo y lugar. A medida que las actividades logísticas logren cumplir con las exigencias del donde y cuando lo quieren los clientes, en igual medida la empresa se diferenciará de la competencia.

En este contexto, Porter (2015) establece que para identificar las actividades de valor es necesario determinar los elementos de diferenciación existentes y potenciales; y explotar el proceso en sus componentes primarios y de apoyo. Tal como lo sugiere López (2019), se debe descomponer el proceso logístico en su forma básica, a través de cinco elementos, los cuales abarcan según el mismo autor lo siguiente:

- Servicio al cliente: Es la satisfacción real de necesidades y cumplimiento real de la promesa de servicio.

- Inventarios: Es el mantenimiento de niveles apropiados del producto, para satisfacer la demanda y los niveles de servicio a los clientes.

- Suministros: Es el mantenimiento de los recursos necesarios para satisfacer la política de inventarios.

- Almacenamiento: Busca mantener niveles físicos reales de productos, suministros y recursos en general.

- Transporte y distribución: Componente del sistema que lleva los suministros, productos y recursos necesarios a manos del cliente final.

Por su parte, Serra (2007) define cada una de estas actividades de la manera siguiente:

1. Actividades fundamentales: Asociadas necesariamente a cualquier canal logístico, y resultan primordiales para la efectividad de las funciones logísticas. A saber:

- Procesamiento de pedidos: Es la actividad que origina el movimiento de los productos y el cumplimiento con los servicios solicitados a la empresa, teniendo una gran incidencia en el tiempo del ciclo del pedido realizado por el cliente.

- Gestión de inventarios: Actividad que tiene por objetivo fundamental realizar la gestión para proporcionar la requerida disponibilidad de los bienes que solicita la demanda.

- Transporte: Actividad indispensable en cualquier organización, para poder trasladar los materiales o bienes a los clientes. También se conoce como distribución.

- Servicio al cliente: Provee la respuesta necesaria para todas las actividades del proceso logístico para atender de forma oportuna los requerimientos del cliente. 
2. Actividades de apoyo: Aunque en algún caso puedan tener tanta relevancia como las primeras, a veces no son consideradas por la gestión logística de la empresa, sobre todo en los inicios del desarrollo de la función logística, y son:

- Compras: Permite la selección de los proveedores, cantidades a adquirir y el momento adecuado para realizar las adquisiciones, así como la planeación de los productos. Ésta impacta fundamentalmente en la gestión de inventario.

- Embalaje: Se establece en consideración a los requisitos de tratamiento de los materiales para el transporte, almacenaje o nivel de protección que se debe dar a los materiales para evitar su deterioro o pérdidas. Impacta fundamentalmente en el almacenamiento de los productos.

- Almacenaje: Se relaciona con la determinación del espacio requerido, diseño y arreglo de los almacenes, así como el acomodo de los productos en su interior, añadiendo valor al producto en el tiempo, asociado a la custodia y salvaguarda de sus condiciones.

- Planificación de productos: Sirve de apoyo al procesamiento de pedidos.

- Tratamiento de mercancías: Implica la selección adecuada del equipo de manipulación de materiales en el almacén, así como los detalles de preparación de los pedidos y devolución de productos defectuosos.

- Gestión de la información: Da soporte a todo el sistema logístico, haciendo énfasis en la planificación y control de necesidades.

Para los fines de este estudio, debido a la aplicación a las empresas del sector floricultor, aunado al enfoque de la investigación cuyo énfasis es en las actividades de valor, donde en conformidad con la teoría desarrolla por Porter (2015) debe darse énfasis a aquellas actividades principales para el proceso organizacional, se trabajó básicamente con las corrientes teóricas de López (2019) y Serra (2007), las cuales correlacionadas definen de forma amplia las actividades que integran el proceso logístico, clasificándolas en fundamentales y de apoyo, según el grado de importancia en las funciones logísticas y el valor para los clientes.

\section{Metodología}

El estudio se tipificó como descriptivo, no experimental y transversal (Arias, 2006; Hernández, Fernández y Baptista, 2014). La información fue recolectada a través de dos cuestionarios, uno para los 23 clientes internos del proceso logístico, compuesto por 50 preguntas y el segundo con 60 preguntas dirigido a los 33 trabajadores del área logística de las empresas estudiadas. Los cuestionarios fueron diseñados con preguntas de estimación, quedando validados todos por el juicio de cinco expertos. Se aplicó un censo a las tres poblaciones con el fin de recabar la información, debido al tamaño reducido de la misma.

Para validar la confiabilidad de los resultados, así como la consistencia interna del instrumento de recolección de información utilizado, se recurrió al Alfa de Cronbach. 
Este indicador es una media estadística que permite estimar la fiabilidad de un instrumento de medida a través de un conjunto de ítems de pregunta (Quero, 2010). Para el instrumento aplicado a los clientes del proceso logístico, se obtuvo un Alfa de 0.797 y para el aplicado a los trabajadores del área logística de las empresas del sector floricultor estudiadas se obtuvo un Alfa de 0.877. Si bien no hay consenso respecto al umbral desde el cuál se considera que un instrumento es confiable, (Quero, 2010) indica que valores superiores a 0.8 son satisfactorios, mientras que autores como (Tavakol \& Dennick, 2011) toman como representativo un 0.7. Es posible observar que los coeficientes son representativos en cuanto a la consistencia del instrumento, lo que demuestra que es confiable para describir, cuantificar e identificar las características evaluadas con las preguntas.

\section{Resultados}

Considerando el enfoque de González (2009) los generadores de valor se utilizan cuando la base de la estrategia es la diferenciación del producto o servicio para ser competitivo. Estos factores tienen una particular incidencia sobre el valor generado en las diferentes actividades a lo largo de la cadena de valor. El análisis consiste en identificar los generadores de valor aplicables a cada etapa de la cadena de valor, especialmente de las actividades primarias. Sin embargo, el almacenaje a pesar de ser clasificada por Serra (2007) como una actividad de apoyo, González (2009) la define como básica para la logística empresarial y por ello es necesario identificar los generadores o factores que impulsan el valor en la misma.

Tomando los planteamientos anteriormente expuestos, se consideró para efectos de esta investigación el almacenamiento como actividad de apoyo, debido a la naturaleza de las empresas objeto de estudio, las cuales almacenan sus materiales e insumos básicos utilizados en sus operaciones, para su posterior entrega a los clientes internos. Sin embargo, es necesario determinar en esta actividad los elementos que impulsan o generan valor debido al papel principal que juega en la provisión de un adecuado nivel de servicio al cliente.

Para González (2009) y Paredes-Chacín (2017), la mayoría de las organizaciones emplean herramientas para la medición del desempeño centrados en valores cuantitativos. Sin embargo, la competitividad no sólo puede ser medida en términos económicos (Paredes-Chacín, 2017), ya que este enfoque comienza a transformarse para dar paso a nuevos paradigmas, considerando ahora: nivel de satisfacción del cliente, calidad, eficiencia, entre otros; que al final se traducen en la generación de valor a los clientes.

Todo ello hace suponer según lo señalan González (2009) y Fuentes, Rada y Torrenegra (2016) que el nuevo contexto empresarial permite entender que el éxito de una organización, no sólo es una consecuencia natural de realizar bien las acciones operativas de apoyo, sino que además es necesario generar valor en cada actividad de la empresa para diferenciarse de la competencia y mantener a los clientes en el tiempo.

En este sentido, en la Tabla 1 se establecieron los factores que impulsan el valor en las actividades del proceso logístico, ajustados con las actividades logísticas de apoyo establecidas en la correlación de las teorías de Serra (2007) y González (2009). 
TABLA 1.

Generadores de valor en las actividades logísticas del proceso logístico

\begin{tabular}{|c|c|c|}
\hline $\begin{array}{l}\text { Actividades } \\
\text { del proceso } \\
\text { logístico }\end{array}$ & $\begin{array}{l}\text { Actividades logísticas } \\
\text { según la teoría de } \\
\text { Serra (2007) }\end{array}$ & Generadores \\
\hline Suministros & $\begin{array}{l}\text { Procesamiento de } \\
\text { pedido }\end{array}$ & $\begin{array}{l}\text { Encuesta de atención a proveedores } \\
\text { Existencia de métodos para la recepción de pedidos } \\
\text { Seguimiento a la trayectoria de pedidos } \\
\text { Nivel de servicio por pedido y centro } \\
\text { Flexibilidad para la atención de proveedores } \\
\text { Errores de previsión de demanda } \\
\text { Cumplimiento con los plazos de envío } \\
\text { Progreso en los objetivos de mejoramiento de suministros }\end{array}$ \\
\hline Inventarios & $\begin{array}{l}\text { Gestión de } \\
\text { Inventarios }\end{array}$ & $\begin{array}{l}\text { Precisión al pagar a proveedores } \\
\text { Costo medio de orden de compra } \\
\text { Falta de inventario disponible } \\
\text { Nivel de intercambio de información con los proveedores } \\
\text { Exactitud con los pronósticos } \\
\text { Tiempo de respuesta a los cambios de requerimientos de los } \\
\text { clientes } \\
\text { Número de empleados entrenados por día para tender a los } \\
\text { clientes } \\
\text { Número de empleados entrenados por día para tender a los } \\
\text { proveedores }\end{array}$ \\
\hline Almacenaje & Almacenaje & $\begin{array}{l}\text { Costo por falta de inventario } \\
\text { Costo de almacenamiento } \\
\text { Queja de los proveedores por atención en almacén } \\
\text { Números de incidentes por seguridad en el almacén } \\
\text { Grado de utilización de la capacidad instalada } \\
\text { Productividad referente a entradas en el almacén } \\
\text { Productividad referente a salidas en el almacén } \\
\text { Número de empleados entrenados por día para atender el } \\
\text { almacén }\end{array}$ \\
\hline Transporte & Transporte & $\begin{array}{l}\text { Costo de transporte unitario } \\
\text { Costo de transporte del inventario } \\
\text { Entregas a tiempo } \\
\text { Número de entregas sin errores } \\
\text { Errores en embarques } \\
\text { Número de envíos por pedidos } \\
\text { Número de envíos urgentes a los clientes } \\
\text { Número de empleados entrenados por día para atender el } \\
\text { transporte }\end{array}$ \\
\hline $\begin{array}{l}\text { Servicio al } \\
\text { Cliente }\end{array}$ & Servicio al Cliente & $\begin{array}{l}\text { Costos de atención al cliente } \\
\text { Percepción del servicio por parte del cliente } \\
\text { Tiempo de respuesta a los requerimientos de los clientes } \\
\text { Encuestas de servicio al cliente } \\
\text { Flexibilidad para la entrega a clientes } \\
\text { Quejas de los clientes } \\
\text { Satisfacción total del cliente } \\
\text { Nivel de intercambio de información con los clientes }\end{array}$ \\
\hline
\end{tabular}

Fuente: Adaptación de Gonzalez (2009) y Serra (2007). 
En la Tabla 2 se muestra la opinión del personal logístico y clientes con relación a los factores que impulsan el valor en las actividades fundamentales del proceso logístico, en la misma, se evidencia las respuestas mayormente seleccionada por la población objeto del estudio.

TABLA 2.

Factores que impulsan valor según opinión del personal logístico y clientes

\begin{tabular}{|c|c|c|c|}
\hline $\begin{array}{l}\text { Actividades del } \\
\text { Proceso Logístico }\end{array}$ & Generadores de valor & $\begin{array}{l}\text { Moda - Generadores } \\
\text { de valor según el } \\
\text { personal logístico } \\
\text { de las empresas del } \\
\text { sector floricultor }\end{array}$ & $\begin{array}{c}\text { Moda - } \\
\text { Generadores de } \\
\text { valor según los } \\
\text { clientes de las } \\
\text { empresas del } \\
\text { sector floricultor }\end{array}$ \\
\hline \multirow{7}{*}{$\begin{array}{l}\text { Procesamiento de } \\
\text { Pedidos }\end{array}$} & $\begin{array}{l}\text { Aplicar encuestas de servicio al } \\
\text { cliente }\end{array}$ & 3 & 2 \\
\hline & $\begin{array}{l}\text { Existencia de métodos } \\
\text { apropiados para la recepción de } \\
\text { pedidos }\end{array}$ & 1 & 3 \\
\hline & $\begin{array}{l}\text { Seguimiento a la trayectoria de } \\
\text { los pedidos }\end{array}$ & 2 & 3 \\
\hline & $\begin{array}{l}\text { Nivel de servicio por pedido y } \\
\text { centro }\end{array}$ & 2 & 2 \\
\hline & $\begin{array}{l}\text { Flexibilidad para la atención de } \\
\text { proveedores }\end{array}$ & 3 & 3 \\
\hline & $\begin{array}{l}\text { Errores de previsión de } \\
\text { demanda }\end{array}$ & 2 & 2 \\
\hline & $\begin{array}{l}\text { Cumplimiento con los plazos de } \\
\text { envíos }\end{array}$ & 2 & 1 \\
\hline \multirow{9}{*}{$\begin{array}{l}\text { Gestión de } \\
\text { Inventarios }\end{array}$} & $\begin{array}{l}\text { Progreso en los objetivos de } \\
\text { mejoramiento de suministros }\end{array}$ & 3 & 3 \\
\hline & $\begin{array}{l}\text { Precisión al pagar a } \\
\text { proveedores }\end{array}$ & 2 & 2 \\
\hline & $\begin{array}{l}\text { Costo medio en las órdenes de } \\
\text { compra }\end{array}$ & 1 & 1 \\
\hline & Falta de inventario disponible & 1 & 3 \\
\hline & $\begin{array}{l}\text { Nivel de intercambio de } \\
\text { información con los proveedores }\end{array}$ & 2 & 2 \\
\hline & $\begin{array}{l}\text { Exactitud en los pronósticos de } \\
\text { requerimiento de materiales } \\
\text { por parte del cliente }\end{array}$ & 2 & 2 \\
\hline & $\begin{array}{l}\text { Tiempo de repuesta a los } \\
\text { cambios de requerimientos de } \\
\text { los clientes }\end{array}$ & 1 & 1 \\
\hline & $\begin{array}{l}\text { Número de empleados } \\
\text { entrenados por mes para } \\
\text { atender a los clientes }\end{array}$ & 1 & 2 \\
\hline & $\begin{array}{l}\text { Número de empleados } \\
\text { entrenados por mes para } \\
\text { atender a los proveedores }\end{array}$ & 3 & 3 \\
\hline
\end{tabular}




\begin{tabular}{|c|c|c|c|}
\hline $\begin{array}{l}\text { Actividades del } \\
\text { Proceso Logístico }\end{array}$ & Generadores de valor & $\begin{array}{l}\text { Moda - Generadores } \\
\text { de valor según el } \\
\text { personal logístico } \\
\text { de las empresas del } \\
\text { sector floricultor }\end{array}$ & $\begin{array}{c}\text { Moda - } \\
\text { Generadores de } \\
\text { valor según los } \\
\text { clientes de las } \\
\text { empresas del } \\
\text { sector floricultor }\end{array}$ \\
\hline \multirow{8}{*}{ Almacenamiento } & Costo por perdida de materiales & 2 & 2 \\
\hline & Costo de almacenamiento & 3 & 3 \\
\hline & $\begin{array}{l}\text { Queja de los proveedores por } \\
\text { atención en almacén }\end{array}$ & 2 & 4 \\
\hline & $\begin{array}{l}\text { Número de incidentes de } \\
\text { seguridad en el almacén }\end{array}$ & 2 & 2 \\
\hline & $\begin{array}{l}\text { Grado de utilización de la } \\
\text { capacidad instalada }\end{array}$ & 2 & 3 \\
\hline & $\begin{array}{l}\text { Productividad referente a } \\
\text { entradas en el almacén }\end{array}$ & 2 & 4 \\
\hline & $\begin{array}{l}\text { Productividad referente a } \\
\text { salidas en el almacén }\end{array}$ & 1 & 3 \\
\hline & $\begin{array}{l}\text { Número de empleados } \\
\text { entrenados por mes para } \\
\text { atender el almacenamiento }\end{array}$ & 3 & 3 \\
\hline \multirow{8}{*}{ Transporte } & Costo de transporte unitario & 1 & 1 \\
\hline & $\begin{array}{l}\text { Costo de transporte por tipo de } \\
\text { vehículo }\end{array}$ & 1 & 1 \\
\hline & Entregas a tiempo & 1 & 1 \\
\hline & Numero de entregas sin errores & 2 & 2 \\
\hline & Errores en embarques & 2 & 4 \\
\hline & Numero de envíos por pedido & 2 & 2 \\
\hline & $\begin{array}{l}\text { Numero de envíos urgentes a } \\
\text { los clientes }\end{array}$ & 4 & 4 \\
\hline & $\begin{array}{l}\text { Número de empleados por mes } \\
\text { para atender el transporte }\end{array}$ & 3 & 3 \\
\hline \multirow{8}{*}{ Servicio al cliente } & Costo de atención al cliente & 1 & 3 \\
\hline & $\begin{array}{l}\text { Flexibilidad para la entrega a } \\
\text { clientes }\end{array}$ & 2 & 1 \\
\hline & $\begin{array}{l}\text { Nivel de intercambio de } \\
\text { información con los clientes }\end{array}$ & 3 & 1 \\
\hline & $\begin{array}{l}\text { Tiempo de respuesta a los } \\
\text { requerimientos de los clientes }\end{array}$ & 2 & 1 \\
\hline & Encuestas de servicio al cliente & 4 & 2 \\
\hline & $\begin{array}{l}\text { Percepción del servicio por } \\
\text { parte del cliente }\end{array}$ & 1 & 1 \\
\hline & Satisfacción total del cliente & 1 & 1 \\
\hline & Queja de los clientes & 2 & 1 \\
\hline
\end{tabular}

Fuente: Elaboración propia. 
Donde cada opinión seleccionada se ve en la Tabla 3:

TABLA 3.

Correspondencia de opinión

\begin{tabular}{ll}
\hline \multicolumn{1}{c}{ Opciones (Opiniones) } & Correspondencia \\
\hline Completamente de acuerdo & 1 \\
De acuerdo & 2 \\
Desacuerdo & 3 \\
Completamente en desacuerdo & 4 \\
No sabe & 5 \\
\hline
\end{tabular}

Fuente: Elaboración propia.

Se decidió para el análisis de los resultados tomar como valor estadístico referencial la moda, la cual, según Box, Hunter, \& Hunter (2008) es el valor que cuenta con una mayor frecuencia de selección dentro de una distribución de datos. Sobre esta consideración y tomando sólo aquellas opciones donde el valor modal obtuvo las dos mayores calificaciones, es decir; completamente de acuerdo y de acuerdo. De esta manera, en el Tabla 4 se muestran los elementos que impulsan el valor o generadores de valor según la opinión del personal logístico.

TABLA 4.

Generadores de valor de las actividades del proceso logístico según el personal de las empresas del sector floricultor

\begin{tabular}{ll}
\hline $\begin{array}{c}\text { Actividades del } \\
\text { Proceso logístico }\end{array}$ & \multicolumn{1}{c}{ Generadores de Valor } \\
\hline Procesamiento de pedidos & $\begin{array}{l}\text { La existencia de métodos para la recepción de } \\
\text { pedidos }\end{array}$ \\
$\cdot$ & El seguimiento a la trayectoria de los pedidos \\
$\cdot$ & Monitorear el nivel de servicio por pedido y centro \\
$\cdot$ & Controlar los errores de previsión de la demanda \\
$\cdot$ & El cumplimiento con los plazos de envío \\
$\cdot$ & La precisión al pagar a proveedores \\
$\cdot$ & Controlar el costo medio de orden de compra \\
$\cdot$ & Controlar la falta de inventario disponible \\
$\cdot$ & El nivel de intercambio de información con los \\
& proveedores \\
$\cdot$ & La exactitud en los pronósticos \\
$\cdot$ & El tiempo de respuesta a los cambios de \\
requerimientos de los clientes & El número de empleados entrenados por mes para \\
Gestión de inventarios & atender a los clientes \\
\hline
\end{tabular}




\begin{tabular}{|c|c|}
\hline $\begin{array}{l}\text { Actividades del } \\
\text { Proceso logístico }\end{array}$ & Generadores de Valor \\
\hline \multirow{6}{*}{ Almacenaje } & Controlar los costos por falta de inventario \\
\hline & Monitorear las quejas de los proveedores \\
\hline & Controlar el número de incidentes de seguridad \\
\hline & El grado de utilización de la capacidad instalada \\
\hline & $\begin{array}{l}\text { La productividad referente a atención de entradas en } \\
\text { el almacén }\end{array}$ \\
\hline & $\begin{array}{l}\text { La productividad referente a atención de salidas en } \\
\text { el almacén }\end{array}$ \\
\hline \multirow{6}{*}{ Transporte } & $\begin{array}{l}\text { Monitorear el costo de incremento en cada pieza por } \\
\text { transporte }\end{array}$ \\
\hline & $\begin{array}{l}\text { Monitorear el costo de transporte por tipo de } \\
\text { vehículo }\end{array}$ \\
\hline & Las entregas a tiempo \\
\hline & El número de entregas sin errores \\
\hline & Controlar la cantidad de errores por embarques \\
\hline & $\begin{array}{l}\text { El número de envíos por pedido adicionales y no } \\
\text { previstos }\end{array}$ \\
\hline \multirow{5}{*}{ Servicio al cliente } & Controlar el costo de atención al cliente \\
\hline & $\begin{array}{l}\text { La flexibilidad para la entrega de bienes y servicios } \\
\text { a los clientes }\end{array}$ \\
\hline & $\begin{array}{l}\text { Monitorear el tiempo de respuesta a los } \\
\text { requerimientos de los clientes }\end{array}$ \\
\hline & La percepción del servicio por parte del cliente \\
\hline & La satisfacción total de los clientes \\
\hline
\end{tabular}

Fuente: Elaboración propia.

En la Tabla 4 puede verse que para el procesamiento de pedidos, es importante la existencia de métodos para la recepción de pedidos para disponer de los insumos a tiempo, el seguimiento a la trayectoria de los pedidos para garantizar tiempo de respuesta efectivos, monitorear el nivel de servicio, controlar los errores de previsión de la demanda para no afectar a los clientes por indisponibilidad de productos y el cumplimiento de plazos de envío a los clientes para evitar retrasos en la ejecución de las operaciones.

En el caso de la gestión de inventarios, el personal logístico consideró como elementos generadores de valor: la precisión al pagar proveedores para evitar retrasos en la ejecución de contratos; controlar el costo medio por orden de compra para aprovechar la economía de escala; controlar la falta de inventario disponible para evitar retrasos en la ejecución de las operaciones de las empresas; el nivel de intercambio de información con los proveedores a fin de garantizar el cumplimiento de los plazos de entrega y ejecución de obras y servicios; la exactitud en los pronósticos para sincronizar la demanda con la planificación de inventarios; el tiempo de res- 
puesta a los cambios requeridos por los clientes, es decir, flexibilidad en la función logística y mantener empleados capacitados para la atención de los clientes.

Para el almacenaje, el personal logístico señaló como elementos generadores de valor, el control de los costos por falta de inventario, es decir, el impacto económico que acarrea no contar con el nivel de inventario optimo; monitorear las quejas de los proveedores para resolver inconvenientes que puedan afectar la ejecución de las operaciones; el grado de utilización de la capacidad instalada para disponer de espacios y condiciones adecuadas para la recepción y entrega de productos; la productividad referente a atención de entradas y salidas en el almacén para mantener el nivel de servicio adecuado a las necesidades de los clientes.

Para la actividad del transporte, el personal logístico indicó que las entregas a tiempo y sin errores son elementos que inciden en la generación de valor. Asimismo, consideran que darle flexibilidad a la actividad de transporte permite generar valor a los clientes, pues con ello es posible atener requerimientos de urgencia o no previstos.

Finalmente, el personal logístico considera que controlar los costos la atención al cliente genera valor, pues disminuye los costos efectivos totales de las operaciones y por ende la salud financiera de la empresa. Por otro lado, la flexibilidad en la entrega de bienes y servicios, así como los tiempos de respuesta son elementos de generación de valor, ya que inciden directamente en las operaciones que realizan los clientes internos. Del mismo modo, en la Tabla 5 se muestran los elementos que impulsan el valor o generadores de valor según los clientes de las empresas floricultoras.

Una vez obtenidos los resultados para los clientes y personal logístico, se realizó la comparación entre las opiniones de ambos, con la finalidad de establecer los puntos de encuentro y diferencias existentes.

TABLA 5.

Generadores de valor de las actividades del proceso logístico según los clientes de las empresas del sector floricultor

\begin{tabular}{ll}
\hline Actividades del Proceso logístico & \multicolumn{1}{c}{ Generadores de Valor } \\
\hline & La precisión al pagar a proveedores \\
& Controlar el costo medio de orden de compra \\
& El nivel de intercambio de información con los proveedores \\
& La exactitud en los pronósticos \\
Gestión de inventarios & El tiempo de respuesta a los cambios de requerimientos de los \\
& clientes \\
& El número de empleados entrenados por mes para atender a los \\
& clientes \\
& Aplicar encuestas de servicio al cliente \\
& Monitorear el nivel de servicio por pedido y centro \\
Controlar los errores de previsión de la demanda & El cumplimiento con los plazos de envío \\
\hline
\end{tabular}




\begin{tabular}{ll}
\hline Actividades del Proceso logístico & \multicolumn{1}{c}{ Generadores de Valor } \\
\hline Almacenaje & Controlar los costos por falta de inventario \\
& Controlar el número de incidentes de seguridad \\
\hline & Monitorear el costo de incremento en cada pieza por transporte \\
& Monitorear el costo de transporte por tipo de vehículo \\
Transporte & Las entregas a tiempo \\
& El número de entregas sin errores \\
& El número de envíos por pedido adicionales y no previstos \\
& La flexibilidad para la entrega de bienes y servicios \\
& El nivel de intercambio de información con los clientes \\
& Monitorear el tiempo de respuesta a los requerimientos de los clientes \\
& Aplicar encuestas de servicio al cliente \\
& La percepción del servicio por parte del cliente \\
La satisfacción total de los clientes & La atención a las quejas de los clientes \\
\end{tabular}

Fuente: Elaboración propia.

TABLA 6.

Comparación entre los generadores de valor del proceso logístico según los clientes

y personal logístico de las empresas del sector floricultor del Departamento de Cundinamarca

\begin{tabular}{|c|c|c|c|}
\hline $\begin{array}{l}\text { Actividades del } \\
\text { Proceso logístico }\end{array}$ & $\begin{array}{c}\text { Generadores de Valor } \\
\text { según González (2009) }\end{array}$ & $\begin{array}{l}\text { Generadores de Valor } \\
\text { según los Clientes }\end{array}$ & $\begin{array}{c}\text { Generadores de Valor } \\
\text { según el Personal } \\
\text { Logístico }\end{array}$ \\
\hline $\begin{array}{l}\text { Gestión de } \\
\text { inventarios }\end{array}$ & $\begin{array}{l}\text { Precisión al pagar a } \\
\text { proveedores } \\
\text { Costo medio de orden de } \\
\text { compra } \\
\text { Falta de inventario } \\
\text { disponible } \\
\text { Nivel de intercambio } \\
\text { de información con los } \\
\text { proveedores } \\
\text { Exactitud en los } \\
\text { pronósticos } \\
\text { Tiempo de respuesta } \\
\text { a los cambios de } \\
\text { requerimientos de los } \\
\text { clientes } \\
\text { Número de empleados } \\
\text { entrenados por día para } \\
\text { atender a los clientes } \\
\text { Número de empleados } \\
\text { entrenados por día } \\
\text { para atender a los } \\
\text { proveedores }\end{array}$ & $\begin{array}{l}\text { La precisión al pagar a } \\
\text { proveedores } \\
\text { Controlar el costo medio } \\
\text { de orden de compra } \\
\text { El nivel de intercambio } \\
\text { de información con los } \\
\text { proveedores } \\
\text { La exactitud en los } \\
\text { pronósticos } \\
\text { El tiempo de respuesta a los } \\
\text { cambios de requerimientos de } \\
\text { los clientes } \\
\text { El número de empleados } \\
\text { entrenados por mes para atender } \\
\text { a los clientes }\end{array}$ & $\begin{array}{l}\text { La precisión al pagar a } \\
\text { proveedores } \\
\text { Controlar el costo medio de } \\
\text { orden de compra } \\
\text { Controlar la falta de } \\
\text { inventario disponible } \\
\text { El nivel de intercambio } \\
\text { de información con los } \\
\text { proveedores } \\
\text { La exactitud en los } \\
\text { pronósticos } \\
\text { El tiempo de respuesta } \\
\text { a los cambios de } \\
\text { requerimientos de los } \\
\text { clientes } \\
\text { El número de empleados } \\
\text { entrenados por mes } \\
\text { para atender a los } \\
\text { clientes }\end{array}$ \\
\hline
\end{tabular}




\begin{tabular}{|c|c|c|c|}
\hline $\begin{array}{l}\text { Actividades del } \\
\text { Proceso logístico }\end{array}$ & $\begin{array}{l}\text { Generadores de Valor } \\
\text { según González (2009) }\end{array}$ & $\begin{array}{l}\text { Generadores de Valor } \\
\text { según los Clientes }\end{array}$ & $\begin{array}{c}\text { Generadores de Valor } \\
\text { según el Personal } \\
\text { Logístico }\end{array}$ \\
\hline $\begin{array}{l}\text { Procesamiento de } \\
\text { pedidos }\end{array}$ & $\begin{array}{l}\text { Encuesta de atención a } \\
\text { proveedores } \\
\text { Existencia de métodos } \\
\text { para la recepción de } \\
\text { pedidos } \\
\text { Seguimiento a la } \\
\text { trayectoria de los } \\
\text { pedidos } \\
\text { Nivel de servicio por } \\
\text { pedido y centro } \\
\text { Flexibilidad para la } \\
\text { atención de proveedores } \\
\text { Errores de previsión de } \\
\text { demanda } \\
\text { Cumplimiento con los } \\
\text { plazos de envíos } \\
\text { Progreso en los objetivos } \\
\text { de mejoramiento de } \\
\text { suministros }\end{array}$ & $\begin{array}{l}\text { Aplicar encuestas de } \\
\text { servicio al cliente } \\
\text { Monitorear el nivel de } \\
\text { servicio por pedido y } \\
\text { centro } \\
\text { Controlar los errores de } \\
\text { previsión de la demanda } \\
\text { El cumplimiento con los } \\
\text { plazos de envío }\end{array}$ & $\begin{array}{l}\text { La existencia de } \\
\text { métodos para la } \\
\text { recepción de pedidos } \\
\text { El seguimiento a la } \\
\text { trayectoria de los } \\
\text { pedidos } \\
\text { Monitorear el nivel de } \\
\text { servicio por pedido y } \\
\text { centro } \\
\text { Controlar los errores de } \\
\text { previsión de la demanda } \\
\text { El cumplimiento con los } \\
\text { plazos de envío }\end{array}$ \\
\hline Almacenaje & $\begin{array}{l}\text { Costo por falta de } \\
\text { inventario } \\
\text { Costo de } \\
\text { almacenamiento } \\
\text { Queja de los proveedores } \\
\text { por atención en almacén } \\
\text { Número de incidentes de } \\
\text { seguridad en el almacén } \\
\text { Grado de utilización de } \\
\text { la capacidad instalada } \\
\text { Productividad referente } \\
\text { a entradas en el almacén } \\
\text { Productividad referente } \\
\text { a salidas en el almacén } \\
\text { Número de empleados } \\
\text { entrenados por día } \\
\text { para atender el } \\
\text { almacenamiento }\end{array}$ & $\begin{array}{l}\text { Controlar los costos por } \\
\text { pérdida de materiales } \\
\text { Controlar el número de } \\
\text { incidentes de seguridad }\end{array}$ & $\begin{array}{l}\text { Controlar los costos por } \\
\text { falta de inventario } \\
\text { Monitorear las quejas } \\
\text { de los proveedores } \\
\text { Controlar el número de } \\
\text { incidentes de seguridad } \\
\text { El grado de utilización } \\
\text { de la capacidad } \\
\text { instalada } \\
\text { La productividad } \\
\text { referente a atención de } \\
\text { entradas en el almacén } \\
\text { La productividad } \\
\text { referente a atención de } \\
\text { salidas en el almacén }\end{array}$ \\
\hline Transporte & $\begin{array}{l}\text { Costo de transporte } \\
\text { unitario } \\
\text { Costo de transporte del } \\
\text { inventario } \\
\text { Entregas a tiempo } \\
\text { Número de entregas sin } \\
\text { errores } \\
\text { Errores en embarques } \\
\text { Número de envíos por } \\
\text { pedido } \\
\text { Número de envíos } \\
\text { urgentes a los clientes } \\
\text { Número de empleados } \\
\text { entrenados por día para } \\
\text { atender el transporte }\end{array}$ & $\begin{array}{l}\text { Monitorear el costo de } \\
\text { incremento en cada pieza } \\
\text { por transporte } \\
\text { Monitorear el costo de } \\
\text { transporte por tipo de } \\
\text { vehículo } \\
\text { Las entregas a tiempo } \\
\text { El número de entregas } \\
\text { sin errores } \\
\text { El número de envíos por } \\
\text { pedido adicionales y no } \\
\text { previstos }\end{array}$ & $\begin{array}{l}\text { Monitorear el costo de } \\
\text { incremento en cada } \\
\text { pieza por transporte } \\
\text { Monitorear el costo de } \\
\text { transporte por tipo de } \\
\text { vehículo } \\
\text { Las entregas a tiempo } \\
\text { El número de entregas } \\
\text { sin errores } \\
\text { Controlar la cantidad de } \\
\text { errores por embarques } \\
\text { El número de envíos por } \\
\text { pedido adicionales y no } \\
\text { previstos }\end{array}$ \\
\hline
\end{tabular}




\begin{tabular}{|c|c|c|c|}
\hline $\begin{array}{l}\text { Actividades del } \\
\text { Proceso logístico }\end{array}$ & $\begin{array}{c}\text { Generadores de Valor } \\
\text { según González (2009) }\end{array}$ & $\begin{array}{l}\text { Generadores de Valor } \\
\text { según los Clientes }\end{array}$ & $\begin{array}{c}\text { Generadores de Valor } \\
\text { según el Personal } \\
\text { Logístico }\end{array}$ \\
\hline Servicio al cliente & $\begin{array}{l}\text { Costo de atención al } \\
\text { cliente } \\
\text { Percepción del servicio } \\
\text { por parte del cliente } \\
\text { Tiempo de respuesta a } \\
\text { los requerimientos de los } \\
\text { clientes } \\
\text { Encuestas de servicio al } \\
\text { cliente } \\
\text { Flexibilidad para la } \\
\text { entrega a clientes } \\
\text { Queja de los clientes } \\
\text { Satisfacción total del } \\
\text { cliente } \\
\text { Nivel de intercambio } \\
\text { de información con los } \\
\text { clientes }\end{array}$ & $\begin{array}{l}\text { La flexibilidad para } \\
\text { la entrega de bienes y } \\
\text { servicios } \\
\text { El nivel de intercambio } \\
\text { de información con los } \\
\text { clientes } \\
\text { Monitorear el tiempo } \\
\text { de respuesta a los } \\
\text { requerimientos de los } \\
\text { clientes } \\
\text { Aplicar encuestas de } \\
\text { servicio al cliente } \\
\text { La percepción del servicio } \\
\text { por parte del cliente } \\
\text { La satisfacción total de } \\
\text { los clientes } \\
\text { La atención a las quejas } \\
\text { de los clientes }\end{array}$ & $\begin{array}{l}\text { Controlar el costo de } \\
\text { atención al cliente } \\
\text { La flexibilidad para } \\
\text { la entrega de bienes y } \\
\text { servicios a los clientes } \\
\text { Monitorear el tiempo } \\
\text { de respuesta a los } \\
\text { requerimientos de los } \\
\text { clientes } \\
\text { La percepción del } \\
\text { servicio por parte del } \\
\text { cliente } \\
\text { La satisfacción total de los } \\
\text { clientes }\end{array}$ \\
\hline
\end{tabular}

Fuente: Elaboración propia.

En la Tabla 6 se muestran los generadores de valor indicados por los clientes y personal logístico de las empresas del sector floricultor del Departamento de Cundinamarca. La información que sustenta dicho cuadro fue extraída de la Tabla 5 que muestra los generadores de valor del proceso logístico según los clientes y la Tabla 4, que muestra los generadores de valor para dicho proceso según la opinión del personal logístico.

Como puede verse en la Tabla 6, para el procesamiento de pedidos los clientes señalaron que la aplicación de encuestas de atención, monitorear el nivel de servicio, controlar los errores de previsión de demanda y cumplir con los plazos de envío; son factores que generan valor a las actividades del proceso logístico en las empresas del sector floricultor del Departamento de Cundinamarca. Por su parte, el personal del área logística comparte la visión de los clientes y a su vez, consideran otros factores de valor como la existencia de métodos para la recepción de pedidos y el seguimiento a la trayectoria de pedidos.

Asimismo, la aplicación de encuestas de atención a los proveedores, el progreso en los objetivos de mejoramiento de suministros y la flexibilidad para la atención de proveedores no fueron consideradas por las poblaciones objeto de estudio como generadores de valor.

De igual manera, los clientes y personal logístico coincidieron en señalar que los generadores de valor para actividad referente a la gestión de inventarios y compras son: la precisión al pagar a proveedores, el control del costo medio de orden de compra, el nivel de intercambio de información con los proveedores, la exactitud en los pronósticos, el tiempo de respuesta a los cambios de requerimientos de los clientes y número de empleados entrenados por mes para atender a los clientes. 
Sin embargo, el personal logístico considera adicionalmente que controlar la falta de inventario disponible también es un generador de valor de dicha actividad. Cabe destacar, que el número de empleados entrenados por día para atender los proveedores no fue considerado por las poblaciones objeto de estudio como un factor que genera valor a la gestión de inventarios.

Asimismo, para la actividad referente al almacenaje las poblaciones objeto de estudio consideran que controlar los costos por pérdida de materiales y el número de incidentes de seguridad son factores que generan valor en dicha actividad. No obstante, el personal logístico señaló que atender las quejas de los proveedores, aprovechar la capacidad instalada para almacenamiento y la atención de las entradas y salidas del almacén, son otros factores que impulsan el valor de dicha actividad.

Por otra parte, los clientes y trabajadores de las áreas logísticas señalaron según los resultados obtenidos que el número de empleados entrenados por día para atender el almacenamiento y los costos de almacenamiento no son generadores de valor.

En cuanto a la actividad de transporte, tanto los clientes como el personal logístico consideran fundamental monitorear el costo de transporte y el incremento de este por cada pieza añadida. También, las entregas a tiempo y el número de entregas sin errores son factores que, según ambos participantes del proceso logístico, generan valor al transporte. Adicionalmente, los clientes señalaron que otro factor que impulsa el valor en esta actividad son los envíos por pedido adicionales y no previstos.

Del mismo modo, el personal logístico consideró el control de errores por embarques como otro factor que agrega valor. Es importante señalar que, para ambas poblaciones, el número de envíos urgentes a los clientes y el número de empleados entrenados por día para atender el transporte no son factores que impulsen el valor en el transporte.

Por último, los clientes y personal logístico coinciden en señalar que la flexibilidad para la entrega de bienes y servicio, la percepción del servicio por parte del cliente, el monitoreo del tiempo de respuesta a los requerimientos de los clientes y la satisfacción total de los mismos son generadores de valor de la actividad de servicio al cliente.

Por su parte, los clientes señalaron adicionalmente que la atención a las quejas de los clientes, el nivel de intercambio de información y la aplicación de encuestas de servicio a estos últimos son generadores de valor dicha actividad. Igualmente, el personal logístico considera que otro factor que impulsa el valor es el control de los costos de atención a los clientes.

Considerando los planteamientos anteriores, y dándole mayor relevancia a los señalamientos de los clientes por ser estos la razón de ser de la logística empresarial, en la Tabla 7 se muestran los generadores de valor del proceso logístico de las empresas del sector floricultor del departamento de Cundinamarca (Colombia). 
TABLA 7.

Generadores de valor del proceso logístico de las empresas del sector floricultor del Departamento de Cundinamarca

\begin{tabular}{|c|c|}
\hline $\begin{array}{l}\text { Actividades del } \\
\text { Proceso Logístico }\end{array}$ & Generadores de Valor \\
\hline Gestión de inventarios & $\begin{array}{l}\text { La precisión al pagar a proveedores } \\
\text { Controlar el costo medio de orden de compra } \\
\text { El nivel de intercambio de información con los proveedores } \\
\text { La exactitud en los pronósticos } \\
\text { El tiempo de respuesta a los cambios de requerimientos de } \\
\text { los clientes } \\
\text { El número de empleados entrenados por mes para atender a } \\
\text { los clientes }\end{array}$ \\
\hline Procesamiento de pedidos & $\begin{array}{l}\text { Aplicar encuestas de servicio al cliente } \\
\text { Monitorear el nivel de servicio por pedido y centro } \\
\text { Controlar los errores de previsión de la demanda } \\
\text { El cumplimiento con los plazos de envío }\end{array}$ \\
\hline Almacenaje & $\begin{array}{l}\text { Controlar los costos por pérdida de materiales } \\
\text { Controlar el número de incidentes de seguridad }\end{array}$ \\
\hline Transporte & $\begin{array}{l}\text { Monitorear el costo de incremento en cada pieza por } \\
\text { transporte } \\
\text { Monitorear el costo de transporte por tipo de vehículo } \\
\text { Las entregas a tiempo } \\
\text { El número de entregas sin errores } \\
\text { El número de envíos por pedido adicionales y no previstos }\end{array}$ \\
\hline Servicio al cliente & $\begin{array}{l}\text { La flexibilidad para la entrega de bienes y servicios } \\
\text { El nivel de intercambio de información con los clientes } \\
\text { Monitorear el tiempo de respuesta a los requerimientos de } \\
\text { los clientes } \\
\text { Aplicar encuestas de servicio al cliente } \\
\text { La percepción del servicio por parte del cliente } \\
\text { La satisfacción total de los clientes } \\
\text { La atención a las quejas de los clientes }\end{array}$ \\
\hline
\end{tabular}

Fuente: Elaboración propia.

En el caso de las empresas del sector floricultor del Departamento de Cundinamarca, se establecieron como generadores de valor en la gestión de inventarios: la precisión al pagar proveedores para evitar retrasos en la ejecución de contratos; el control del costo medio por orden de compras para obtener insumos a precios competitivos, a través de economía de escala; el nivel de intercambio de información con los proveedores para sincronizar las operaciones de los proveedores y la empresa, de tal manera que se garantice la ejecución de obras y servicios en las condiciones contratadas.

Así mismo, la exactitud en los pronósticos para sincronizar la demanda con la planificación de productos, monitorear los tiempos de respuesta para atender a los clientes internos según sus necesidades; y el número de empleados entrenados para atender a los clientes internos. 
En la actividad de procesamiento de pedido, las empresas del sector floricultor estudiadas requieren la aplicación de encuestas de servicio para conocer aspectos de interés en los clientes internos de tal manera que se agregue valor a la función logística; monitorear el nivel de servicio para satisfacer las necesidades de los clientes internos; controlar los errores de la previsión de demanda para sincronizar la planificación de productos y lo requerido por los clientes internos; y el cumplimiento con los plazos de envío de materiales e insumos para evitar retrasos en la ejecución de las operaciones de la empresa, y por ende la continuidad en la prestación del servicio de su actividad económica.

Para el almacenaje, se consideraron como elementos generadores de valor: el control de los costos por pérdida de materiales para mantener una salud financiera adecuada en las empresas floricultoras; y controlar el número de incidentes de seguridad para evitar daños humanos que conduzcan al cese temporal de operaciones en las empresas objeto de estudio.

Para la actividad del transporte, las entregas a tiempo y sin errores son elementos que inciden en la generación de valor. Asimismo, consideran que darle flexibilidad a la actividad de transporte permite generar valor a los clientes, pues con ello es posible atener requerimientos de urgencia o no previstos.

Finalmente, es necesario que las empresas floricultoras ofrezcan flexibilidad en la entrega de bienes y servicios, monitoreen los tiempos de respuesta y mantengan un nivel de intercambio de información adecuado con los clientes internos, son elementos de generación de valor, ya que inciden directamente en las operaciones que estos realizan.

\section{Conclusiones}

Se logró identificar los factores que generan valor en las actividades del proceso logístico. Mismos que son definidos como factores que tienen particular incidencia sobre el valor generado en las diferentes actividades a lo largo de la cadena de valor y son la base para establecer diferenciación entre la empresa y la competencia.

El éxito de una organización no solo es consecuencia lógica de realizar bien las acciones operativas de apoyo, sino que además es necesario generar valor en cada actividad de la empresa para satisfacer los requerimientos de los clientes y mantenerlos en el tiempo. Por lo tanto, se recomienda el uso de indicadores, aparte de los financieros, que permitan identificar los factores que generan valor en los procesos, tal como lo expresa González (2009).

Partiendo de los principios teóricos de González (2009), se consultó la opinión de los clientes y personal logístico del sector floricultor objeto de estudio, lo cual permitió determinar los generadores de valor, según la perspectiva de cada población estudiada. 
La comparación entre los resultados obtenidos entre clientes y personal de las empresas objeto de estudio, permitió evidenciar que la mayoría de los factores que generan valor en las actividades del proceso logístico son coincidentes entre ambos grupos. Sin embargo, existen otros factores que fueron señalados por los clientes pero que para los trabajadores no impulsan el valor en las actividades, y viceversa.

Finalmente, se determinaron los generadores de valor que deben ser considerados por las empresas floricultoras para crear un impacto y satisfacción en sus clientes, a través de su proceso logístico más claro. En virtud a lo anterior, se proporciona un conocimiento que puede ser útil y referente para otras empresas en sectores iguales o diferentes al estudiado.

\section{Reconocimientos y AgRadecimientos}

Los autores Wilfrido Arteaga y Abraham González agradecen a la Universidad Militar Nueva Granada y a la Vicerrectoría de Investigación por el apoyo en la elaboración del presente artículo.

El autor José Ignacio Rodríguez agradece a la Universidad Distrital Francisco José de Caldas y a la Vicerrectoría de Investigación por el apoyo en la elaboración del presente artículo.

Artículo derivado del proyecto de investigación titulado "Cadena de valor del proceso logístico de las empresas floricultoras del Departamento de Cundinamarca".

\section{REFERENCIAS}

Arias, F. (2006). El Proyecto de Investigación. Introducción a la metodología cientifica. Caracas: Episteme.

Bowersox, D., Cross, D., \& Cooper, M. (2007). Administración y Logística en la Cadena de Suministro. México, D.F.: McGraw Hill.

Box, G., Hunter, J., \& Hunter, W. (2008). Estadística para investigadores. (2a $E d$.). Barcelona: Reverté.

Casanovas, A., \& Cuatrecasas, L. (2003). Logística Empresarial: Gestión Integral de la Información y Material en la Empresa. Barcelona: Ediciones Gestión 2000 S.A.

Chumaceiro, A., Chirinos, E. \& Reyes, I. (2016). History of political and social organizations aimed at promoting corporate social responsibility in the twentieth century. Búsqueda, 3(16), 20-32. https://doi.org/10.21892/01239813.163

Fuentes, G., Rada, J. y Torrenegra, A. (2016). Medición de variables psicológicas, económicas y sociales para identificar factores ocultos de los emprendedores en Barranquilla. Económicas CUC, 37(1), 177-202. http://dx.doi. org/10.17981/econcuc.15.5.2016.08

González, A. (2009). Actividades de Valor Logística como soporte para la Medición del Desempeño de los Elementos Éticos en las Empresas del Sector Eléctrico. [Tesis doctoral]. Maracaibo: Universidad Rafael Belloso Chacín. 
Hernández, R., Fernández, C. \& Baptista, P. (2014). Metodología de la investigación. (6a Ed.). México, D.F.: McGraw-Hill.

López, D. (2019). Gestión Logística: Herramienta Indispensable para la Competitividad. [Online]. https://www.gestiopolis.com/gestion-logistica-competitividad-empresarial/

Paredes-Chacín, J. (2017). Planificación financiera ante la perspectiva organizacional en empresas cementeras del estado Zulia-Venezuela. Económicas CUC, 38(1), 105-132. https://doi.org/10.17981/econcuc.38.1.05

Porter, M. (2015). Estrategias competitivas. Técnicas para el análisis de los sectores industriales. México, D.F.: Grupo Editorial Patria.

Quero, M. (2010). Confiabilidad y coeficiente Alpha de Cronbach. Telos. Revista de Estudios Interdiciplinarios en Ciencias Sociales, 12(2), 248-252. Disponible en http://ojs.urbe.edu/index.php/telos/article/view/2416

Ramírez, J., Valero, G. y Martínez, P. (2019). Oportunidades de las Minicadenas Productivas del Sector Cacao de Santander Frente al Pos Conflicto Colombiano. Económicas CUC, 40(2), 153-182. https://doi.org/10.17981/ econcuc.40.2.2019.10

Serra, D. (2007). La Logística Empresarial en el Nuevo Milenio. Barcelona: Gestión 2000.

Tavakol, M. \& Dennick, R. (2011). Making sense of Cronbach's alpha. International Journal of Medical Education, 2, 53-55. https://oi.org/10.5116/ ijme.4dfb.8dfd

\section{BIODATA}

Wilfrido Javier Arteaga Sarmiento es Ingeniero Industrial (2003) de la Universidad del Atlántico (Colombia) con Master en Gestión del Conocimiento y la Documentación (2013) de la Universidad Montpellier 3 (Francia) y Magister en Ingeniería Industrial (2019) de la Universidad Distrital Francisco José de Caldas (Colombia). Profesor Asistente de la Universidad Militar Nueva Granada (Colombia) desde 2017. Investigador en las áreas de mejoramiento de procesos, logística y creación de valor empresarial. https://orcid.org/0000-00017890-7751

\footnotetext{
Abraham Jesús González es Ingeniero Industrial (1999) con Master en Gerencia de Empresas mención Mercadeo (2005) de la Universidad del Zulia (Venezuela). Doctorado en Ciencias Gerenciales (2009) de la Universidad Dr. Rafael Belloso Chacín (Venezuela). Certificado como Auditor Interno de Calidad ISO 9001:2008 por FONDONORMA. Profesor Asociado de la Universidad Militar Nueva Granada (Colombia) desde 2017. Investigador en las áreas de mejoramiento de procesos, logística y creación de valor empresarial. https:// orcid.org/0000-0002-3298-1902
} 
José Ignacio Rodríguez Molano es Ingeniero Industrial (1998) y Magister ciencias de la información y las comunicaciones (2012) de la Universidad Distrital Francisco José de Caldas (Colombia). Doctorado En Ingeniería Informática (2017) de la Universidad de Oviedo (España). Profesor Asociado del Programa de Ingeniería Industrial de la Universidad Distrital Francisco José de Caldas (Colombia). https://orcid.org/0000-0003-2581-277X 